\title{
Avaliação do comportamento em fadiga de juntas estruturais de ligas de Al2024T3 coladas com adesivo epóxi
}

\author{
MARCHEZIN, E. ${ }^{\text {I }}$ PARDINI, L.C. .I, GUIMARÃES,V.A. ${ }^{\text {II }}$ \\ I Instituto Tecnológico de Aeronáutica - ITA, Praça Marechal Eduardo Gomes, 50, Vila das Acácias, \\ CEP 12228-900, São José dos Campos, SP, Brasil \\ e-mail: eduardo.marchezin@gmail.com \\ ${ }^{\mathrm{II}}$ Divisão de Materiais - AMR/IAE/CTA, São José dos Campos, SP, Brasil \\ e-mail: pardini@iae.cta.br

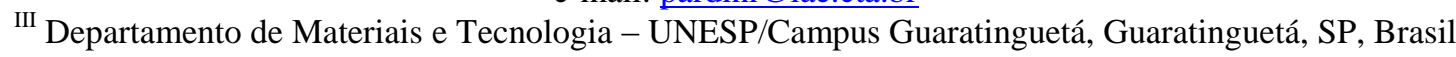 \\ e-mail: valdir@feg.unesp.br
}

\begin{abstract}
RESUMO
Ligas de alumínio são extensamente usadas em partes aeronáuticas devido às boas propriedades mecânicas e baixa densidade. Estas partes devem ser unidas para formar conjuntos maiores. Uma junta estrutural é definida como um segmento de estrutura que provê um meio de transferir carga de um elemento estrutural para outro. A maioria das juntas aeronáuticas é mecanicamente fixada com múltiplos prendedores (parafusos ou rebites). Estas juntas apresentam uma alta concentração de tensões ao redor do prendedor, porque a transferência de carga entre elementos da junta acontece em uma fração da área disponível. Por outro lado, as cargas aplicadas em juntas adesivas são distribuídas sobre toda a área colada e reduz os pontos de concentração de tensão. Juntas são a fonte mais comum de falhas estruturais em aeronaves e quase todos os reparos envolvem juntas. Portanto, é importante entender todos os aspectos de projeto e análise de juntas. O objetivo deste trabalho é comparar estaticamente juntas estruturais de ligas de Al2024-T3 em três condições: juntas mecanicamente rebitadas, juntas coladas e uma configuração híbrida rebitada e colada. Foi usada a norma NASM 1312-4 para confecção dos corpos-de-prova. Além disso, foram conduzidos testes de fadiga, sob amplitude de carregamento constante e razão de tensão igual a 0,1 para avaliar a eficiência dos elementos estruturais durante sua vida em serviço. Os resultados mostraram que a configuração híbrida apresenta maior resistência estática e uma vida em fadiga superior à configuração colada.
\end{abstract}

Palavras-chave:Ligas de Al 2024-T3, juntas estruturais, fadiga, colagem metálica, junta sobreposta simples.

\section{Evaluation of fatigue behavior of Al2024T3 alloys bonded with epoxy adhesive}

\section{ABSTRACT}

Aluminum alloys are widely used in aeronautical parts due to their good mechanical properties and low densities. These parts must be joined together to form larger assemblies. A structural joint is defined as a segment of structure that provides a means of transferring load from one structural member to another. The majority of aircraft joints are mechanically fastened with multiple fasteners. These joints present a high concentration of stresses around the fastener, because the load transfer between elements of the joint has to take place over a fraction of the available area. By contrast, the applied loads in the adhesive joints are distributed over the entire bonded area and reduce points of stress concentration. Joints are the most common source of structural failures in aircraft and almost all repairs involve joints. Therefore, it is important to understand all aspects of joint design and analysis. The aim of the present work was to perform a comparative evaluation of the structural joints of Al2024-T3 alloy in three conditions: mechanically fastened joint, bonded joints and a hybrid conFiguration of bonded-riveted joint. The Standard Test Method for determining strength properties of mechanically fastened sheet metal lap joints (NASM 1312-4) was used in all specimen conFigurations. Besides, fatigue tests were conducted in specimens under constant amplitude loading at a stress ratio of 0.1 to evaluate the efficiency of the structural elements during their service life. The results showed that when metal-to-metal adhesive bond procedures are used with a mechanically fastened technique, the static strength and fatigue life of the joint will increase.

Keywords: Al 2024-T3 alloy, structural joints, fatigue, metal bonding, single lap joint. 


\section{INTRODUÇÃO}

As diversas partes e componentes que constituem uma aeronave precisam ser unidas para formarem subconjuntos. A união de vários subconjuntos é feita em montagens maiores, que por sua vez são instaladas na aeronave. Estas uniões são feitas através de juntas estruturais que providenciam o meio de transferir carga de um elemento estrutural a outro. Juntas representam um dos maiores desafios no projeto de estruturas em geral. Nos pontos de transferência de força (interfaceamento) ocorrem concentrações de tensões e o elemento de união e/ou as regiões da junta envolvida estão sempre pré-deformados [1]. Para efeitos de projeto estas deformações podem ser tratadas como causadoras de uma tensão residual devida ao processo de fabricação. Além disso, as juntas são a fonte mais comum de falhas estruturais em aeronaves e quase todos os reparos envolvem juntas. Portanto, é importante entender todos os aspectos de projeto e análise de juntas.

A maioria das juntas em aeronaves é mecanicamente unida com múltiplos prendedores (rebites ou parafusos). O mais eficiente uso dos prendedores é feito com uma junta projetada para transferir carga por cisalhamento, conforme ilustra a Figura 1. Entretanto, restrições geométricas algumas vezes requerem que os prendedores sejam carregados em tração []].

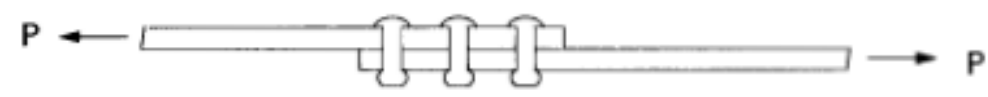

Figura 1: Junta sobreposta simples, rebitada, carregada em cisalhamento.

Independentemente do tipo de carregamento, a zona unida sempre se apresenta com uma deformação inicial (devido à pré-tensão) ou residual (devido ao processo de fabricação). Quando a união é solicitada ocorrem concentrações de tensões nos pontos de transferência de força (interfaceamento), o que determina sobremaneira o comportamento da união sob solicitação em serviço []].

A concentração de tensões em juntas mecânicas rebitadas é particularmente severa porque a transferência de carga entre elementos da junta está concentrada em uma fração da área disponível. Juntas coladas são por sua vez estruturalmente mais eficientes porque providenciam melhores oportunidades de eliminar concentrações de tensões, ao distribuir sobre uma área maior o carregamento da junta [4]], conforme ilustra a Figura $2[\underline{5}]$.

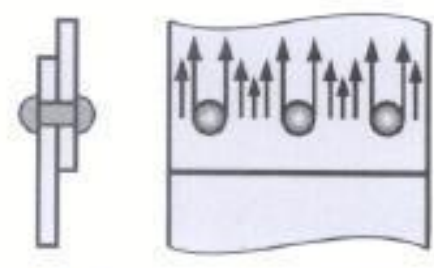

Junta Rebitada

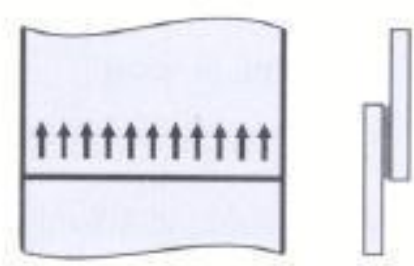

Junta colada

Figura 2: Concentração de tensões em juntas rebitadas e coladas.

Em função dessas características, foi possível observar que existe uma lacuna em aplicações práticas das técnicas de construção de colagem estrutural metálica e indica a oportunidade para desenvolvimento do assunto.

O presente trabalho se propõe a comprovar os prováveis benefícios do uso de juntas coladas, analisar a resistência e durabilidade deste tipo de união e os modos de falha, comparando-os com uma junta tradicional rebitada e uma configuração hibrida colada e rebitada.

\section{FADIGA EM JUNTAS COLADAS E REBITADAS}

De acordo com alguns estudos independentes, entre $70 \%$ e $90 \%$ dos casos de falhas estruturais de componentes ocorre pelo mecanismo de fadiga. Fadiga é definida como o processo que conduz à falha do material pela aplicação repetida de um determinado carregamento, mesmo para tensões bem abaixo da resis- 
tência de ruptura do material. A presença de danos físicos microscópicos ou de defeitos pré-existentes no material pode acumular-se através de uma deformação plástica com contínuos ciclos até desenvolverem-se para uma trinca.

O comportamento à fadiga de juntas coladas difere dos metais em muitos aspectos []]. A durabilidade da junta colada é afetada pelas propriedades do aderente, do adesivo e principalmente pelas interações que ocorrem na interface entre aderente e adesivo [7]. As características de resistência da interface dependem por sua vez da preparação de superfície dos aderentes e da sua capacidade de resistir aos efeitos de temperatura, umidade, envelhecimento e ciclos de carregamento $[\underline{8}, \underline{9}]$.

Os dados de testes realizados pelos fabricantes de adesivos são úteis somente para propósitos comparativos e devem ser usados de maneira restrita pelo projetista. Atualmente, o desenvolvimento de procedimentos de análise de fadiga em juntas coladas é objeto de intensa pesquisa e já existem alguns procedimentos tradicionalmente usados para metais que estão sendo gradativamente adaptados para prever falhas em juntas coladas [10].

A metodologia utilizada para a análise de fadiga no presente trabalho foi o método SN, onde a análise é feita baseando-se em curvas tensão em função da vida em fadiga. O método SN é simples e possui uma série de vantagens, que o torna confiável na maioria dos casos práticos para o dimensionamento mecânico à fadiga [11].

As curvas SN podem ser apresentadas em gráficos do tipo log-log ou linear-log. Curvas com tensão normalizada podem ser obtidas dividindo-se a tensão máxima aplicada durante o ensaio de fadiga pela carga estática de ruptura da junta. A curva normalizada SN para juntas coladas pode ser aproximada pela Equação 1 [12]:

$$
\sigma_{M A X} / \sigma_{U L T}=1-\kappa \log N_{f}
$$

Onde:

- $\sigma$ max é a tensão máxima aplicada - MPa

- $\sigma$ ult é a tensão de ruptura estática - MPa

- $\quad \mathrm{N}_{\mathrm{f}}$ é a ciclagem até a falha - Ciclos

- $\quad \mathrm{k}$ é a perda fracional de resistência por décadas de ciclos

O valor de $\mathrm{k}$ é uma medida da resistência à fadiga da junta e depende da geometria da junta e das condições de carregamento. Quanto menor o valor de $\mathrm{k}$ maior a resistência à fadiga. A Tabela 1 mostra típicos valores de $\mathrm{k}$ para diferentes tipos de juntas coladas com adesivo epóxi [12].

Tabela 1: Típicos valores de $\mathrm{k}$ para juntas coladas $(\mathrm{R}=0,1$ e $\mathrm{f}=5 \mathrm{~Hz})$.

\begin{tabular}{l|c}
\hline \multicolumn{1}{c|}{ Configuração da Junta } & $\mathrm{k}$ \\
\hline Topo com arestas chanfradas em $30^{\circ}$ (aderentes de alumínio) & 0,055 \\
\hline Sobreposta dupla (aderentes de titânio) & 0,075 \\
\hline Cinta dupla (aderentes de alumínio) & 0,088 \\
\hline Sobreposta simples (aderentes de aço) & 0,093 \\
\hline Sobreposta dupla (fibra de vidro) & 0,097 \\
\hline Junta em T (tração direta) & 0,104 \\
\hline Junta em T - rasgamento (aderentes de aço) & 0,130 \\
\hline
\end{tabular}

O desempenho da junta depende da geometria e dos valores de picos de tensões que ocorrem nas extremidades da junta. Por exemplo, uma junta em ângulo $30^{\circ}$ onde a falha é dominada por tensões de cisalhamento tem uma vida em fadiga superior àquelas onde tensões de rasgamento são predominantes [13].

As trincas por fadiga em juntas rebitadas geralmente se iniciam nos furos das últimas carreiras de cravação, onde se encontram os rebites mais carregados. Por este motivo, a determinação da distribuição de tensões ao longo destas carreiras de cravação se faz necessária ao analisar a junta rebitada. Portanto, a informação básica a ser obtida para um projeto de junta rebitada é a quantidade de carga transferida na primeira e na última fileira de rebites. Tensões de esmagamento (bearing) e tensões passantes (bypass) são criadas pela redistribuição do fluxo de carga que ocorre no furo devido à carga transferida pelo rebite. A carga de esma- 
gamento é a força aplicada pelo rebite em seu furo. Carga passante é a força remanescente aplicada na junta que passa para o rebite seguinte. Utilizando o principio do equilíbrio de forças, conclui-se que um acréscimo da tensão de esmagamento está diretamente relacionado a um decréscimo da tensão passante. Se por um lado elevadas tensões de esmagamento favorecem o surgimento de trincas nos furos dos rebites, por outro lado altas tensões passantes aumentam a taxa de propagação de trincas ao longo dos rebites [14].

Dessa forma, para tentar-se quantificar este efeito, utiliza-se normalmente o chamado fator de concentração de tensões, simbolizado normalmente pelo símbolo kt, que nada mais é do que a razão entre a tensão máxima nas vizinhanças da descontinuidade geométrica e alguma tensão de referência, normalmente adotada como a tensão nominal, longe da descontinuidade, ou alguma tensão de referência que considere somente a área líquida (descontando a área da descontinuidade), conhecida como tensão liquida, ou a tensão de esmagamento (bearing stress), etc. O fator de concentração de tensões é calculado pela Equaçã [1드, 16]:

$$
k_{t}=\frac{\sigma}{S}
$$

Onde:

- $\quad \mathrm{k}_{\mathrm{t}}$ é o fator de concentração de tensões

- $\sigma$ é a tensão local na região da descontinuidade - MPa

- $\quad$ S é a tensão nominal ou de referência - MPa

Embora na maioria das vezes as estruturas sejam projetadas para permanecerem no regime elástico, a determinação dos concentradores de tensão é de extrema importância, pois além de complementarem a garantia da não ocorrência de deformação plástica, são utilizados para se quantificar o decréscimo de resistência destes quando submetidos a cargas cíclicas, ou seja, nos problemas de fadiga.

\section{MATERIAIS E MÉTODOS}

No desenvolvimento experimental do trabalho utilizou-se substrato de Al2024-T3. Esta liga de alumínio é largamente usada em aplicações aeronáuticas, pois possui excelente resistência à fadiga e tenacidade à fratura [17]. As peças de alumínio foram fabricadas a partir de uma única chapa de material, seguindo a direção de grão longitudinal, com o objetivo de se evitar possíveis efeitos de anisotropia do material.

Na confecção dos corpos-de-prova rebitados foram utilizados rebites BACR15BB5D5 (rebites sólidos com cabeças protuberantes, norma MS20470), com diâmetro de aproximadamente $4 \mathrm{~mm}$. O material do rebite é a liga $\mathrm{Al}-\mathrm{Cu} 2017-\mathrm{T} 3$ que possui uma resistência ao cisalhamento de $262 \mathrm{MPa}$.

O adesivo epóxi empregado na fabricação dos corpos-de-prova colados apresenta alta resistência mecânica, sendo proveniente da Henkel Corporation. As propriedades físicas e mecânicas do adesivo estrutural Hysol EA 9394 utilizado são mostradas na Tabela 2 [18]. Com o objetivo de eliminar dispersão nos resultados devido à variação na matéria-prima do adesivo, foi utilizado o mesmo lote de material para fabricação de todos os corpos-de-prova colados.

Tabela 2: Propriedades do Adesivo Estrutural EA 9394

\begin{tabular}{l|c}
\hline Propriedades & Valores para EA 9394 \\
\hline Resistência à Tração & $46 \mathrm{MPa}$ \\
\hline Módulo de Elasticidade à Tração & $4,2 \mathrm{GPa}$ \\
\hline Limite máximo de deformação & $1,66 \%$ \\
\hline Resistência ao cisalhamento & $28,9 \mathrm{MPa}$ \\
\hline Módulo de Cisalhamento & $1,02 \mathrm{GPa}$ \\
\hline Densidade da resina curada & $1,36 \mathrm{~g} / \mathrm{ml}$ \\
\hline
\end{tabular}

Para o desenvolvimento dos ensaios de fadiga e tração, foram fabricados trinta e oito corpos-deprova, em três configurações diferentes. As configurações estudadas são: juntas rebitadas, juntas coladas e uma configuração híbrida de junta colada e rebitada. Esses corpos-de-prova foram fabricados de acordo com 
a norma NASM 1312-4 [19], desenvolvidas pela National Aerospace Standard (NAS), conforme ilustra a Figura 3.

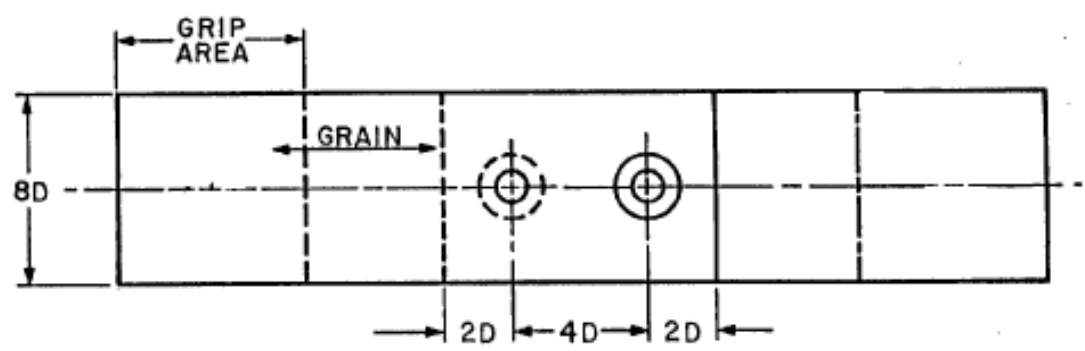

Figura 3: Orientação da Norma NASM 1312-4 para a fabricação dos corpos-de-prova.

As peças de alumínio utilizadas na confecção dos corpos-de-prova colados e híbridos seguiram as mesmas dimensões utilizadas na fabricação dos corpos-de-prova rebitados. A preparação da superfície dos substratos foi realizada de acordo com o trabalho de WEGMAN [20]. A aplicação do adesivo seguiu as recomendações do fabricante do adesivo epóxi.

A aplicação de pressão e temperatura foi controlada computacionalmente utilizando-se um equipamento do tipo hotbonder, modelo HCS9200B, que permite fazer rampas de aquecimento e resfriamento a uma taxa de $5^{\circ} \mathrm{C} / \mathrm{min}$. O ciclo de temperatura escolhido foi de $93^{\circ} \mathrm{C}$ e levando em consideração a rampa de aquecimento e a temperatura ambiente, demora-se cerca de 13 minutos para que se atinja a temperatura desejada. Durante a fase de aquecimento a viscosidade da resina é mínima, porém as reações de intercruzamento já iniciaram. Dessa forma, é importante manter a resina no patamar de $93^{\circ} \mathrm{C}$ por 60 minutos para se evitar a degradação do material por exotermia [21]. Para expulsar o excesso de adesivo e compactação das camadas dos corpos-de-prova foi aplicado vácuo de $71,1 \mathrm{kPa}$ durante todo o processo de cura.

Os corpos-de-prova da configuração híbrida, junta colada e rebitada simultaneamente, foram fabricados mesclando-se os processos descritos anteriormente. Desta forma, optou-se por aplicar uma fina camada de adesivo em ambas às peças, rebitá-las e em seguida submetê-las ao ciclo de cura do adesivo.

Os ensaios de tração e fadiga dos corpos-de-prova do presente trabalho foram feitos utilizando um equipamento de ensaio de tração e compressão para cargas constantes e cíclicas modelo INSTRON 8801, com capacidade para suportar cargas de até 10 ton.

Neste trabalho, o objetivo do ensaio estático de tração até a ruptura foi comparar a resistência de cada uma das diversas configurações de corpos-de-prova e também proporcionar valores de referência para os ensaios de fadiga.

O ensaio de fadiga realizado tem o propósito de comparar através da obtenção da curva SN as propriedades de vida em fadiga de diversas configurações de corpos-de-prova do tipo junta sobreposta ciclicamente carregada, de modo a produzir tensões de cisalhamento na junta.

Neste trabalho, os ensaios de fadiga nas três diferentes configurações de juntas foram feitos com níveis de tensões que variam de $19 \%$ a $96 \%$ da carga estática de ruptura da junta. Além disso, os ensaios foram limitados a 120.000 ciclos, o equivalente a duas vidas econômicas de uma típica aeronave comercial. Os corpos-de-prova que atingiram a marca de 120.000 ciclos foram submetidos posteriormente a um ensaio de tração residual. Outros parâmetros utilizados nos ensaios de fadiga foram uma razão de tensão de 0,1 e uma frequência de ensaio de $10 \mathrm{~Hz}$.

Além disso, uma análise de falha foi efetuada nos corpos de prova ensaiados, a fim de se verificar os mecanismos de falha envolvidos (falhas adesivas, coesivas ou do substrato). De posse dos resultados dos ensaios, foram feitas extrapolações e comparações, com o intuito de validar a efetividade de se usar reforços localizados colados ao invés de rebites, no caso de reparos ou mesmo para substituir uma usinagem química durante a fabricação da estrutura.

\section{RESULTADOS E DISCUSSÕES}

\subsection{Ensaios de Tração}

A Figura 4 mostra o gráfico de força em função do deslocamento para os ensaios de tração dos quatro corpos-de-prova da configuração colada. Nota-se que os corpos-de-prova desta configuração apresentam um comportamento elástico [22]. A média das cargas de ruptura foi de 2.472,99 N, com um deslocamento médio até a ruptura de $0,26 \mathrm{~mm}$. Os corpos-de-prova colados apresentaram modo de falha adesiva. 


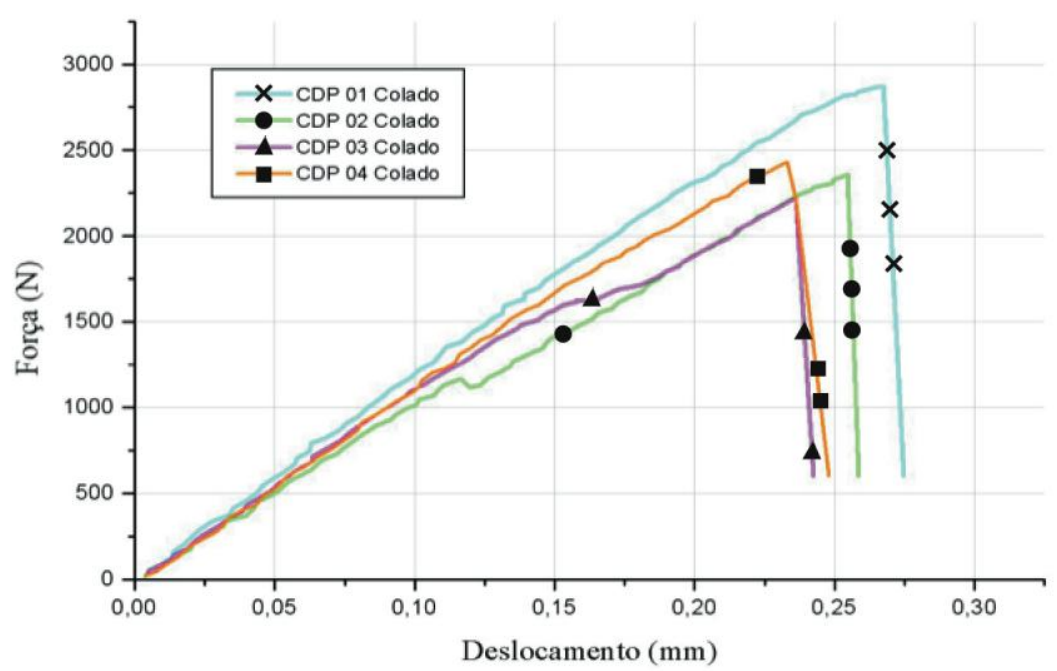

Figura 4: Ensaios de tração em corpos-de-prova colados.

A Figura 5 mostra o gráfico de força em função do deslocamento para os para os ensaios de tração dos quatro corpos-de-prova da configuração rebitada. As juntas rebitadas apresentaram um comportamento elástico-plástico. Observa-se a região de comportamento elástico até uma faixa de valores de $2.000 \mathrm{~N}$ e 0,25 $\mathrm{mm}$ de deslocamento. A média das cargas de ruptura foi de $3 \cdot 660,71 \mathrm{~N}$ e o deslocamento médio até este ponto foi em torno de $1,05 \mathrm{~mm}$. Os corpos-de-prova desta configuração apresentaram modo de falha por esmagamento.

A Figura 6 mostra o gráfico de força em função do deslocamento para o ensaio de tração dos dez corpos-de-prova da configuração híbrida (rebitada e colada). As juntas híbridas também apresentaram um comportamento elástico-plástico. Observa-se no gráfico uma região de comportamento elástico até o primeiro modo de falha da junta - falha adesiva. Neste momento ocorre o rompimento da colagem na junta, caracterizado por um forte ruído durante o ensaio. Em seguida, ocorre uma queda acentuada no carregamento com pouca variação do deslocamento, e a junta assume um comportamento plástico até a ocorrência do segundo modo de falha - falha por esmagamento do furo.

Considerando o valor médio obtido nos ensaios com corpos-de-prova rebitados $(3.660,71 \mathrm{~N})$ como a configuração padrão, temos que a carga média de ruptura da configuração colada $(2.472,99 \mathrm{~N})$ foi $32 \%$ menor. Por outro lado, os corpos-de-prova híbridos $(4.765,73 \mathrm{~N})$ apresentaram um desempenho $30 \%$ superior à configuração rebitada. 


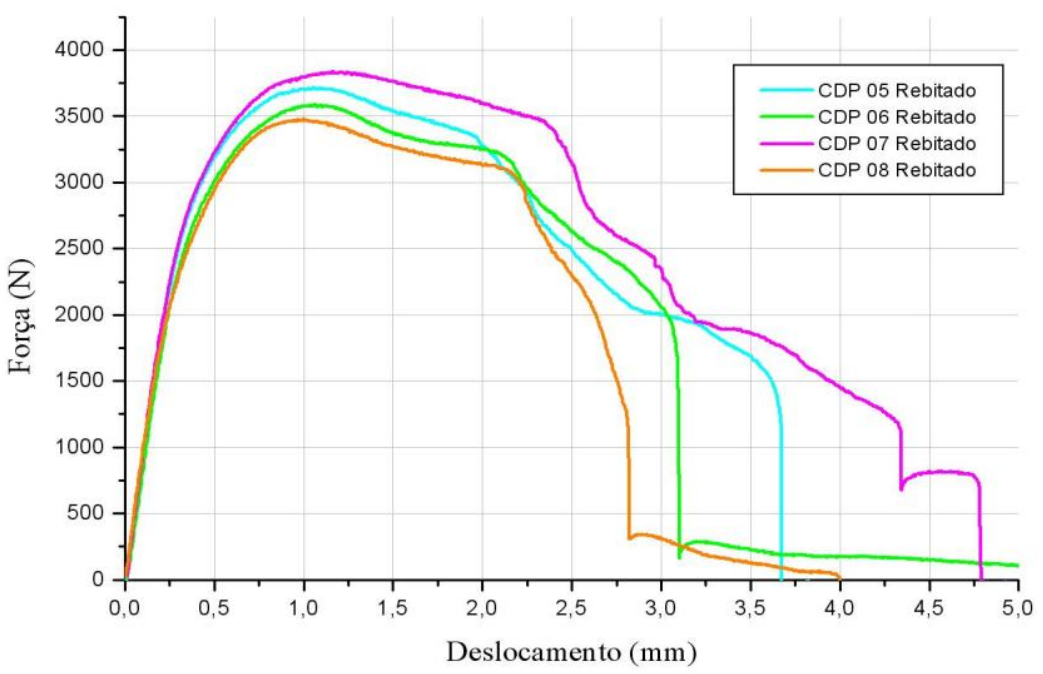

Figura 5: Ensaios de tração em corpos-de-prova rebitados.

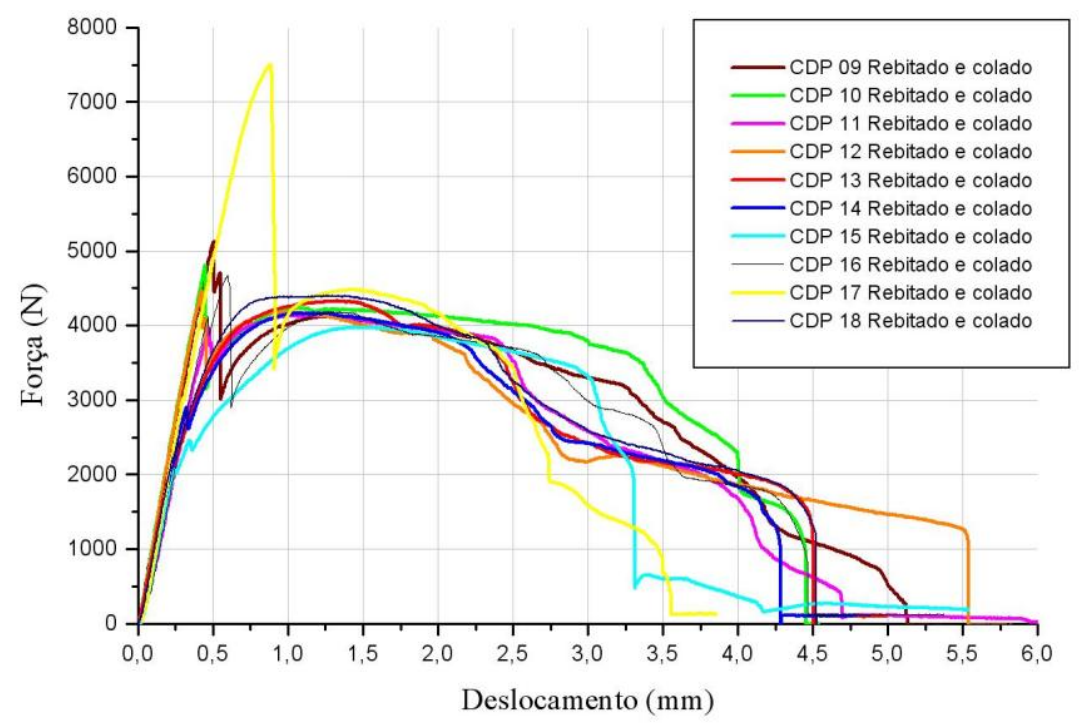

Figura 6: Ensaios de tração em corpos-de-prova híbridos (rebitados e colados).

\subsection{Ensaios de Fadiga}

A Tabela 3 mostra os resultados do número de ciclos (Nf) dos ensaios de fadiga para diferentes níveis de carregamento realizados nos corpos-de-prova colados. A tensão máxima é obtida dividindo-se a carga máxima aplicada pela área de colagem do corpo-de-prova. Além disso, são apresentados os modos de falha, a amplitude do carregamento (A) e os valores máximos (Pmax), médios (Pmed) e mínimos (Pmin) das forças aplicadas. A tensão normalizada ( $\sigma$ mAX $/ \sigma$ uLt) é obtida pelo quociente entre a tensão máxima e a tensão de ruptura estática (ou tensão de ensaio média para corpos-de-prova colados). 
Tabela 3: Resultados dos ensaios de fadiga nos corpos-de-prova colados.

\begin{tabular}{c|c|c|c|c|c|c|c|c}
\hline \# CDP & Tipo & $\sigma_{M A X}(\mathrm{MPa})$ & Pmax (N) & Pmin (N) & A (N) & Pmed (N) & Modo de Falha & $N_{f}($ ciclos $)$ \\
\hline C-05-80 & Colado & 1,89 & $1.930,1$ & 193,0 & 868,5 & 1061,6 & Falha adesiva & 53 \\
\hline C-06-80 & Colado & 0,46 & 458,2 & 45,8 & 206,2 & 252,0 & Falha adesiva & 120.000 \\
\hline C-07-80 & Colado & 1,38 & $1.387,4$ & 138,7 & 624,3 & 763,1 & Falha adesiva & 5.071 \\
\hline C-08-80 & Colado & 1,08 & $1.093,1$ & 109,3 & 491,9 & 601,2 & Falha adesiva & 8.291 \\
\hline C-09-70 & Colado & 0,77 & 770,5 & 77,1 & 346,7 & 423,8 & Falha adesiva & 4.703 \\
\hline C-10-70 & Colado & 0,61 & 613,0 & 61,3 & 275,9 & 337,2 & Falha adesiva & 120.000 \\
\hline C-11-70 & Colado & 0,69 & 702,6 & 70,3 & 316,2 & 386,4 & Falha adesiva & 120.000 \\
\hline C-12-70 & Colado & 0,62 & 625,1 & 62,5 & 281,3 & 343,8 & Falha adesiva & 120.000 \\
\hline
\end{tabular}

A Figura 7 mostra o gráfico da tensão normalizada (a) e tensão máxima (b) em função do número de ciclos para os oito corpos-de-prova da configuração colada. Nota-se a falta de pontos intermediários na curva. Pequenas variações no carregamento podem ser significativas e provocar uma falha com baixos ciclos.

(a)

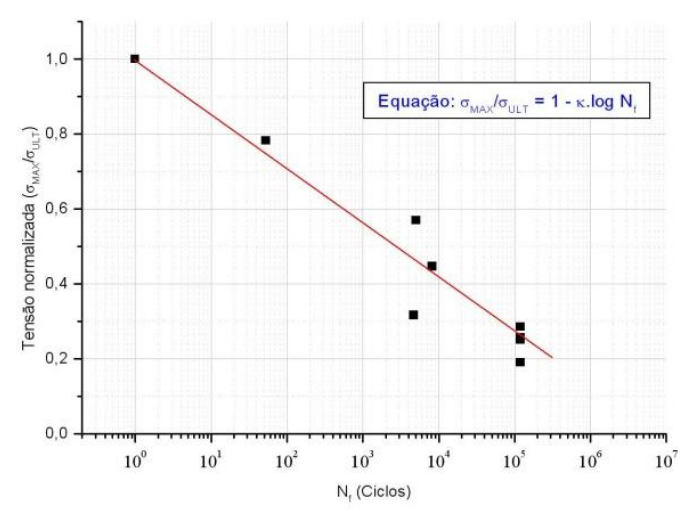

(b)

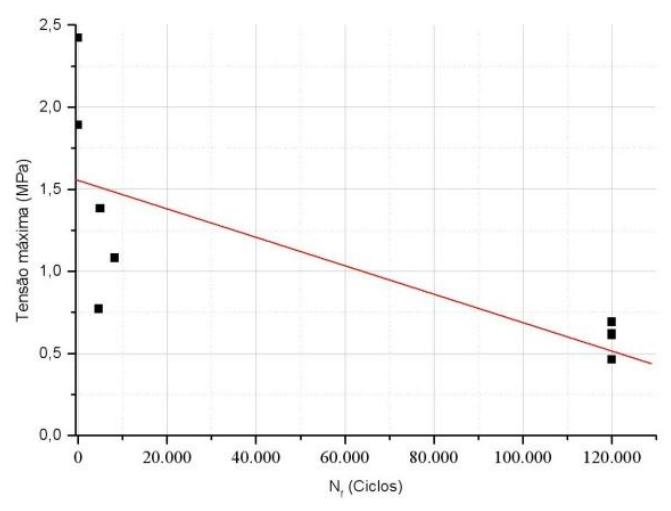

Figura 7: (a) Curva SN para a configuração colada. (b) Gráfico da Tensão máxima x ciclos.

A partir dos resultados experimentais da curva SN apresentados na Figura 7 e com base na Equaçã (1) foi possível determinar o valor da perda fracional de resistência por décadas de ciclos ( $\kappa)$. Para as juntas coladas do presente trabalho, o valor da perda fracional $(\kappa)$ obtido foi de 0,148 . Este resultado é inferior aos valores apresentados na Tabela 1, demonstrando uma menor resistência à fadiga. A geometria da junta escolhida e as condições de carregamento, principalmente as concentrações de tensões nas extremidades da junta, podem simultaneamente ter influenciado neste resultado. A melhoria destas variáveis, com a obtenção de um $\kappa$ intermediário na Tabela 1 (por exemplo, $\kappa=0,093$ ), poderia aumentar a durabilidade da junta colada para 120.000 ciclos com uma tensão normalizada de apenas 0,52, ou seja, 52\% da tensão de ruptura estática, ou então, seria possível atingir o limite de fadiga (um milhão de ciclos) com apenas $44 \%$ da tensão de ruptura estática.

A Tabela 4 mostra os resultados do número de ciclos $(\mathrm{Nf})$ dos ensaios de fadiga para diferentes níveis de carregamento realizados nos corpos-de-prova rebitados. A tensão máxima é a tensão de esmagamento que é obtida pela Equaçã abaixo:

$$
f_{b}=\frac{P}{d \cdot t}
$$


Onde:

- $f_{b}$ é a tensão de esmagamento

- P é a carga aplicada

- t é a espessura da chapa

- d é o diâmetro do rebite

Tabela 4: Resultados dos ensaios de fadiga nos corpos-de-prova rebitados.

\begin{tabular}{c|c|c|c|c|c|c|c|c}
\hline \# CDP & Tipo & $\sigma_{\text {MAX }}(\mathrm{MPa})$ & Pmax (N) & Pmin (N) & A (N) & Pmed (N) & Modo de Falha & \multirow{2}{*}{$($ ciclos $)$} \\
\hline R-05-80 & Rebitado & 650,3 & $2.477,6$ & 247,8 & $1.114,9$ & $1.362,7$ & Esmagamento & 120.000 \\
\hline R-06-80 & Rebitado & 933,6 & $3.408,9$ & 340,9 & $1.534,0$ & $1.874,9$ & Esmagamento & 1.047 \\
\hline R-07-80 & Rebitado & 823,2 & $2.973,1$ & 297,3 & $1.337,9$ & $1.635,2$ & Esmagamento & 76.343 \\
\hline R-08-80 & Rebitado & 739,7 & $2.847,6$ & 284,8 & $1.281,4$ & $1.566,2$ & Esmagamento & 45.034 \\
\hline R-09-70 & Rebitado & 929,6 & $3.283,4$ & 328,3 & $1.477,5$ & $1.805,9$ & Esmagamento & 16.776 \\
\hline R-10-70 & Rebitado & 818,5 & $3.086,2$ & 308,6 & $1.388,8$ & $1.697,4$ & Esmagamento & 43.837 \\
\hline R-11-70 & Rebitado & 871,7 & $3.321,3$ & 332,1 & $1.494,6$ & $1.826,7$ & Esmagamento & 17.377 \\
\hline R-12-70 & Rebitado & 637,4 & $2.352,5$ & 235,3 & $1.058,6$ & $1.293,9$ & Esmagamento & 106.193 \\
\hline
\end{tabular}

A Figura 8 mostra o gráfico da tensão normalizada (a) e tensão máxima (b) em função do número de ciclos para os oito corpos-de-prova da configuração rebitada.

(a)

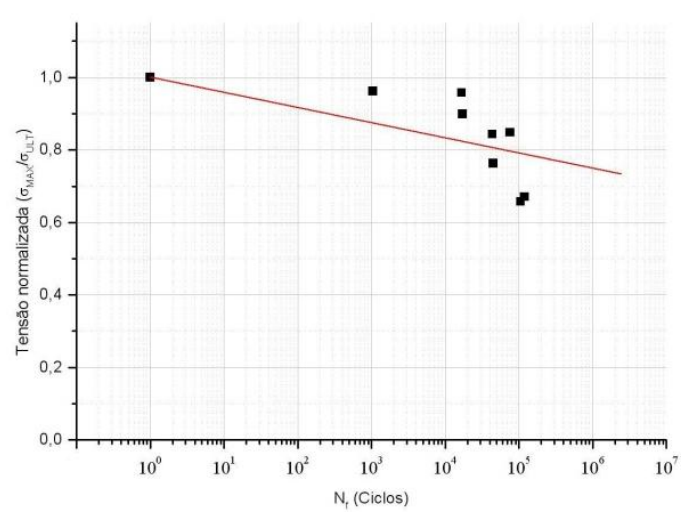

(b)

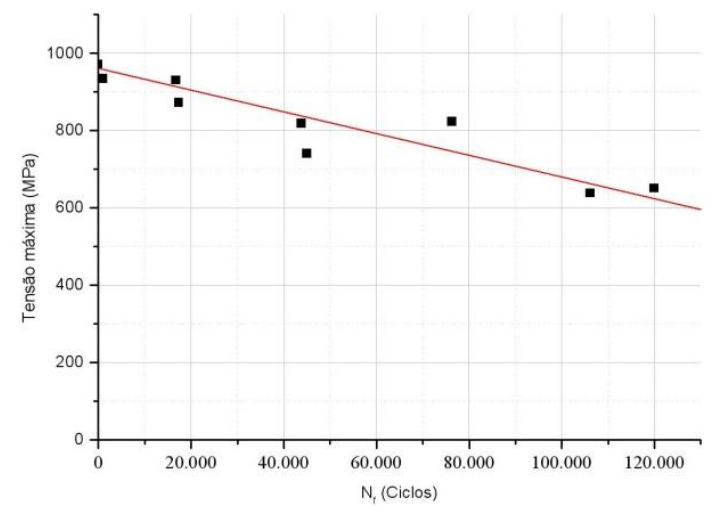

Figura 8: (a) Curva SN para a configuração rebitada. (b) Gráfico da Tensão máxima x ciclos.

Para a determinação dos fatores de entalhe para fadiga dos corpos-de-prova rebitados, foi utilizada a curva SN do material A12024T3, com razão de tensão igual a 0,020 [23]. Foi verificado qual seria o nível de tensão necessário na curva SN para que ocorresse a falha da junta com a quantidade de ciclos obtidos durante o ensaio. Essa tensão determinada a partir da curva foi dividida pela tensão aplicada durante o ensaio, obtendo assim o fator de entalhe para fadiga ( $\mathrm{kf}$ ) dos corpos-de-prova rebitados ensaiados.

Com kf determinado, foi feito o cálculo para determinação do coeficiente de concentração de tensão com $\alpha=0,51$ (alumínio) e $\rho=4,0 \mathrm{~mm}$. A Tabela 5 mostra os fatores de entalhe para fadiga kf e os coeficientes de concentração de tensão kt para os corpos-de-prova que apresentavam pontos tabulados na curva SN. 
Tabela 5: Valores de kf e kt para as juntas rebitadas.

\begin{tabular}{c|c|c|c|c}
\hline \# CDP & Tensão remota (MPa) & Tensão remota (KSI) & $k_{f}$ & $k_{t}$ \\
\hline R-05-80 & 73,73 & 10,70 & 4,58 & 5,04 \\
\hline R-07-80 & 85,29 & 12,37 & 4,37 & 4,79 \\
\hline R-08-80 & 86,56 & 12,55 & 4,58 & 5,04 \\
\hline R-10-70 & 92,96 & 13,48 & 4,34 & 4,77 \\
\hline R-12-70 & 69,19 & 10,04 & 4,93 & 5,43 \\
\hline
\end{tabular}

Pelo resultado apresentado na Tabela 5, pode-se verificar que os coeficientes de concentração de tensão variam entre $\mathrm{k}_{\mathrm{t}}=4,77$ e $\mathrm{k}_{\mathrm{t}}=5,43$ com um valor médio de $\mathrm{k}_{\mathrm{t}}=4,96$.

A Tabela 6 mostra os resultados do número de ciclos $(\mathrm{Nf})$ dos ensaios de fadiga para diferentes níveis de carregamento realizados nos corpos-de-prova híbridos (rebitados e colados). A tensão máxima é obtida dividindo-se a carga máxima aplicada pela área de colagem do corpo-de-prova.

Tabela 6: Resultados dos ensaios de fadiga nos corpos-de-prova híbridos.

\begin{tabular}{|c|c|c|c|c|c|c|c|c|}
\hline \# CDP & Tipo & $\sigma_{M A X}(\mathrm{MPa})$ & $\operatorname{Pmax}(\mathrm{N})$ & $\operatorname{Pmin}(N)$ & $\mathrm{A}(\mathrm{N})$ & Pmed (N) & Modo de Falha & $N_{f}($ ciclos $)$ \\
\hline CR-05-80 & Híbrido & 4,45 & $4,413,2$ & 441,3 & $1.986,0$ & $2.427,3$ & Falha adesiva & 4.391 \\
\hline CR-06-80 & Híbrido & 3,88 & $3.919,8$ & 392,0 & $1.763,9$ & $2.155,9$ & Falha adesiva & 7.831 \\
\hline CR-07-80 & Híbrido & 3,53 & $3.605,1$ & 360,5 & $1.622,3$ & $1.982,8$ & Falha adesiva & 32.097 \\
\hline CR-08-80 & Híbrido & 4,31 & $4.374,6$ & 437,5 & $1.968,6$ & $2.406,0$ & Falha adesiva & 832 \\
\hline CR-09-70 & Híbrido & 2,99 & $3.018,4$ & 301,8 & $1.358,3$ & $1.660,1$ & Falha adesiva & 7.559 \\
\hline CR-10-70 & Híbrido & 4,09 & $4.223,1$ & 422,3 & $1.900,4$ & $2.322,7$ & Falha adesiva & 33 \\
\hline CR-11-70 & Híbrido & 3,68 & $3.727,9$ & 372,8 & $1.677,6$ & $2.050,4$ & Falha adesiva & 13.700 \\
\hline CR-12-70 & Híbrido & 3,18 & $3.219,5$ & 321,9 & $1.448,8$ & $1.770,7$ & Falha adesiva & 18.395 \\
\hline
\end{tabular}

A Figura 9 mostra o gráfico da tensão normalizada (a) e tensão máxima (b) em função do número de ciclos para os oito corpos-de-prova da configuração híbrida. A escolha de níveis elevados de tensões provocou a ocorrência de falhas com baixa ciclagem. 
(a)

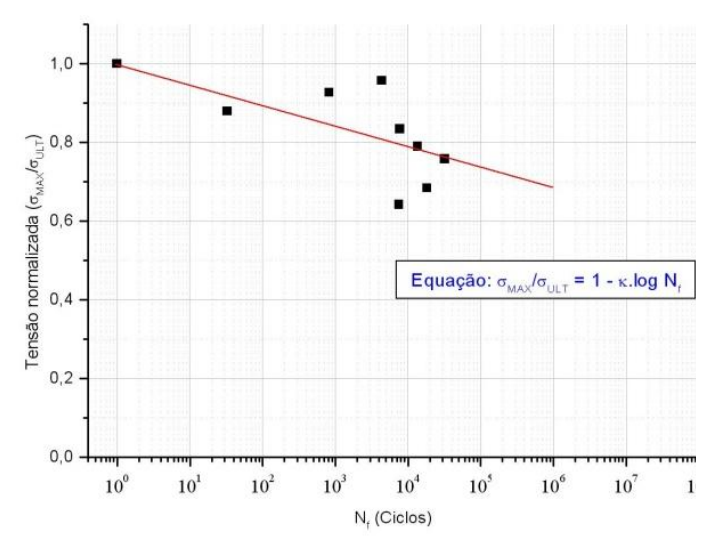

(b)

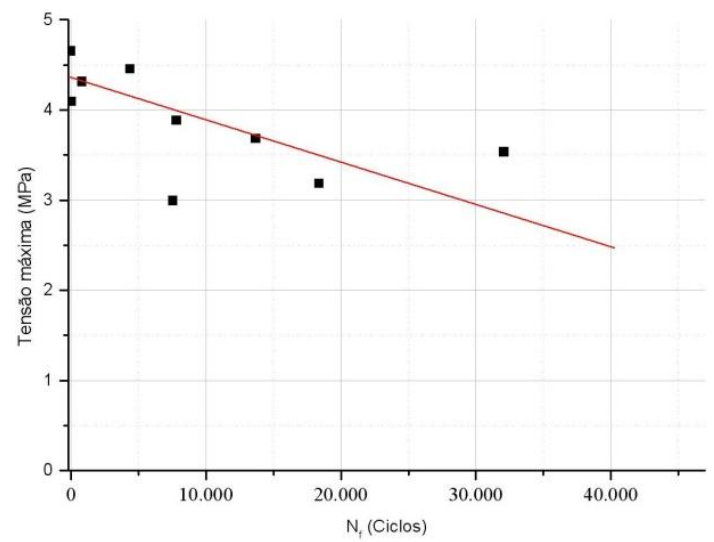

Figura 9: (a) Curva SN para a configuração híbrida. (b) Gráfico da Tensão máxima x ciclos.

\section{CONCLUSÕES}

Considerando o valor médio obtido nos ensaios estáticos de tração com corpos-de-prova rebitados como a configuração padrão, temos que a carga média de ruptura da configuração colada foi $32 \%$ menor. Por outro lado, os corpos-de-prova híbridos apresentaram um desempenho 30\% superior à configuração rebitada.

Para os ensaios dinâmicos, observou-se que os corpos-de-prova colados, com uma tensão máxima aplicada durante o ensaio de fadiga inferior a $30 \%$ da tensão de ruptura estática, apresentaram uma vida em fadiga superior a 120.000 ciclos, o que equivale a duas vidas econômicas de uma típica aeronave comercial.

O baixo valor de perda fracional $(\kappa)$ obtido para a junta colada sugere que a geometria e as condições de carregamento podem ser melhoradas ainda mais, aumentando desta forma a resistência à fadiga da junta.

Os corpos-de-prova híbridos apresentaram uma vida em fadiga média duas vezes maiores do que os corpos-de-prova colados ensaiados nas mesmas condições, mesmo com cargas aplicadas três vezes maiores. A análise dos ensaios dos corpos-de-prova da configuração híbrida mostra que o uso em conjunto de técnicas de colagem e rebitagem tem o potencial de aumentar a carga de trabalho da junta colada com pouco decréscimo da vida em fadiga.

Pode-se concluir que o trabalho apresentou progressos significativos relacionados à avaliação do comportamento em fadiga de juntas estruturais de ligas de Al2024T3 coladas com adesivo epóxi e o objetivo em uma próxima etapa será otimizar a geometria da junta colada, visando aumentar o desempenho em fadiga.

\section{BIBLIOGRAFIA}

[1] HART-SMITH, L.J., "Joints", In: ASM International Handbook Committee - Composites engineering materials handbook, v.1, pp .479-489, 1987.

[2] BRUHN, E.F., “Analysis and design of flight vehicle structures”, EUA, Purdue University, 1973.

[3] COGNARD, P., “Adhesive and sealants - basic concepts and high tech bonding ”, Versailles, France: Elsevier, v. 1, pp. 229-244, 2005.

[4] HART-SMITH, L.J., "The strength of adhesive-bonded single-lap joints”, Santa Monica: Douglas Aircraft Company (IRAD Technical Report Number MDC-J0742), 1970.

[5] MILITARY SPECIFICATIONS/DOD - MIL HDBK 17-F, “Composite materials handbook”, USA: Department of Defense, (v. 3, revision F), 2002.

[6] PETRIE, E.M., “Handbook of adhesives and sealants”, New York, NY, McGraw-Hill, 2000. 
[7] MATTHEWS, F.L., RAWLINGS, R.D., “Composite materials: engineering and science”, Cambridge, England, Woodhead Publishing Limited, 1994.

[8] HOSKIN, B.C., BAKER, A.A., “Composite materials for aircraft structures”, New York, AIAA Educational Series, pp. 237, 1986.

[9] NIU, M.C.Y., “Airframe structural design: practical design information and data”, Hong Kong Conmilit Press Limited, 1988.

[10] HARTSHORN, S.R., "Structural adhesives - chemistry and technology”, New York, NY, Plenum Press, 1986.

[11] KLESNIL, M., LUKÁŠ, P., “Fatigue of metallic materials”, Amsterdam, Elsevier, 2nd edition, 1992.

[12] NPL - NATIONAL PHYSICAL LABORATORY, disponível em: http://www.npl.co.uk/publications/design-requirements-for-bonded-and-bolted-compositestructures. Acessado em janeiro de 2007.

[13] ARMSTRONG, K., BEVAN, L.G., COLE, W.F., "Care and repair of advanced composites”, Warrendale, PA, SAE, 2005.

[14] SWIFT, T., “Repairs to damage tolerant aircraft”, Berlin, Springer-Verlag, pp. 433-483, 1991.

[15] CONWAY, J.B., SJODAHL, L.H., “Analysis and representation of fatigue data”, Materials Park, Ohio, ASM International, 1991.

[16] ROOKE, D.P., CARTWRIGHT, D.J., "Stress intensity factors”, Her Majesty's Stationery Office, Londres, 1974.

[17] MILITARY SPECIFICATIONS/DOD - MMPDS, "Metallic materials properties development and standardization", USA, Department of Defense, 2003.

[18] HENKEL AEROSPACE, disponível em: www.aerospace.henkel.com. Acessado em janeiro de 2009.

[19] NASM 1312-4: Fastener Test Methods Method 4 Lap Joint Shear, USA, Department of Defense, 1997.

[20] WEGMAN, R.F., "Surface preparation techniques for adhesive bonding”, New Jersey, Noyes Publications, 1989.

[21] CARLSSON, L.A., PIPES, B., "Experimental characterization of advanced composite materials", New Jersey, Prentice-Hall, 1987.

[22] COGNARD, P., "Adhesive and sealants - basic concepts and high tech bonding”, Elsevier, v.2, Versailles, France, pp. 227, 2005.

[23] MILITARY SPECIFICATIONS/DOD - MIL HDBK 5H, "Metallic materials and elements for aerospace vehicle structures", USA, pp.3-115, 1998. 